\title{
Road traffic mortality in the Slovak Republic in 1996-2014
}

Alexandra Brazinova MD PhD, Marek Majdan PhD

\section{APPENDIX}

\section{Correspondence}

Department of Public Health Faculty of Health Sciences and Social Work

Trnava University

Univerzitne nam.1

91701 Trnava, Slovak Republic

tel.+421918641974

alexandra.brazinova@gmail.com

alexandra.brazinova@truni.sk

International Neurotrauma Research Organization

Mölker Gasse 4/3

A-1080 Wien, Austria

tel.: +4369910720609

abrazinova@igeh.org 


\section{Slovak Republic - brief country characteristics and list of adopted road safety measures}

Since its establishment in 1993 (after the split of the Czechoslovak Republic), the Slovak Republic developed into a democratic state, and has become a member of the European Union and the North Atlantic Treaty Organization. With regard to road safety, many steps have been taken in the past 20 years in the country: the road infrastructure has grown and its quality has improved, legislation regulating road user behavior and vehicle safety design has been adopted, trauma care has advanced, and the economy of the country has improved. In the field of road safety, the following regulations and other measures have been adopted and have entered force in the Slovak Republic since its establishment (the most significant laws, regulations and other measures taken are included):

- $\quad$ Act 315/1996 Coll. on traffic on surface communications - reinforced rules already in place: zero alcohol tolerance in motor vehicle drivers, mandatory helmet use for motorcycle drivers and passengers, mandatory wearing of seat belt for a person sitting on a seat equipped with a seat belt

- Declaration 265/1996 of the Ministry of Transport, Post and Telecommunications on emission monitoring -setting the emission limits for motor vehicles

- Declaration 116/1997 of the Ministry of Transport, Post and Telecommunications on conditions for traffic on road communications - as of $1^{\text {st }}$ July 1997 motor vehicles must be equipped with headrests for driver and passenger, bus seats must have head rests as well, seatbelts must be installed on all seats of cars for personal use

- Before entering the European Union (2004), the Slovak Republic transposed the European Community legislation into the national legislation. This lead to stricter monitoring of the technical status and emission limits of motor vehicles (Directive no.96/1996 of European Community, adopted in the Slovak Republic in 2005).

- $\quad$ In 2005, the Slovak Republic adopted its first multi-annual Road Safety Plan (2005)

- Government regulation no.554/2006 Coll. on mandatory use of safety belts and child restraints systems (entered into force $1^{\text {st }}$ June 2007) - safety belts became mandatory on all motor vehicle seats.

- In 2009 the Slovak Government subsidized the purchase of new cars to promote the trade-in of old, technically outdated models.

- A New comprehensive law on road traffic was adopted at the end of 2008, and entered into force as of $1^{\text {st }}$ February 2009 as Act No.8/2009 Coll. on the Road Traffic (Road Traffic Act). This act introduced the following changes:

- $\quad$ Motor vehicle headlights must be on at all times of the day and year

- The Speed limit was decreased to from 60 to $50 \mathrm{~km} / \mathrm{hr}$ in town, $90 \mathrm{~km} / \mathrm{h}$ outside town, and $130 \mathrm{~km} / \mathrm{hr}$ on highways

- $\quad$ Cell phone use while driving was prohibited (except hands-free phones)

- $\quad$ Mandatory safe distance between vehicles were enacted 
- Winter tires were declared mandatory in certain climate conditions and/or during the period 15th November- 31st March

- $\quad$ Reflective markings were made mandatory for cyclists and pedestrians

- Helmets for adult bicyclists (15+) on the out-of-town roads, and helmets for childrenbicyclists $<15$ on all roads became mandatory

Sanctions for violation of road traffic regulations were strengthened 
Table A1. Age-standardized mortality rates per 100,000 person years for total population, males and females, individual road user groups, in the Slovak Republic in 19962014. Average mortality rates for road user groups and all road users for the period 1996-2008 (before new road traffic law entering the force) and 2009 -2014 (after the introduction of the law)

\begin{tabular}{|c|c|c|c|c|c|c|c|c|c|c|c|c|c|}
\hline \multicolumn{14}{|c|}{ Mechanism of injury } \\
\hline \multirow[b]{2}{*}{ year } & \multirow[b]{2}{*}{ Total } & \multicolumn{4}{|c|}{ Total } & \multicolumn{4}{|c|}{ Males } & \multicolumn{4}{|c|}{ Females } \\
\hline & & MV & Ped & Bic & $\mathrm{MC}$ & MV & Ped & Bic & $\mathrm{MC}$ & MV & Ped & Bic & MC \\
\hline 1996 & 15,30 & 6,36 & 6,99 & 1,41 & 0,53 & 10,25 & 10,96 & 2,66 & 1,06 & 2,57 & 3,57 & 0,33 & 0,03 \\
\hline 1997 & 18,59 & 8,06 & 8,13 & 1,76 & 0,64 & 13,71 & 13,73 & 3,03 & 1,16 & 2,72 & 3,24 & 0,61 & 0,12 \\
\hline 1998 & 19,40 & 8,71 & 7,32 & 2,46 & 0,92 & 14,28 & 12,22 & 4,65 & 1,70 & 3,35 & 3,03 & 0,71 & 0,17 \\
\hline 1999 & 15,53 & 6,67 & 6,45 & 1,92 & 0,50 & 10,61 & 10,15 & 3,52 & 0,98 & 2,62 & 2,98 & 0,45 & 0,03 \\
\hline 2000 & 14,69 & 5,55 & 6,03 & 2,37 & 0,74 & 9,20 & 10,25 & 3,99 & 1,41 & 2,05 & 2,22 & 0,92 & 0,09 \\
\hline 2001 & 14,04 & 5,83 & 5,68 & 2,02 & 0,52 & 9,51 & 8,96 & 3,73 & 1,00 & 2,22 & 2,62 & 0,54 & 0,03 \\
\hline 2002 & 13,21 & 5,87 & 5,18 & 1,46 & 0,70 & 9,60 & 8,15 & 2,27 & 1,33 & 2,36 & 2,70 & 0,71 & 0,10 \\
\hline 2003 & 14,29 & 6,12 & 5,37 & 2,10 & 0,70 & 9,89 & 8,54 & 3,42 & 1,28 & 2,48 & 2,59 & 1,02 & 0,13 \\
\hline 2004 & 12,99 & 5,64 & 5,00 & 1,55 & 0,80 & 9,30 & 7,84 & 2,95 & 1,60 & 2,19 & 2,57 & 0,38 & 0,00 \\
\hline 2005 & 12,54 & 5,77 & 4,50 & 1,39 & 0,88 & 8,95 & 6,95 & 2,67 & 1,66 & 2,76 & 2,36 & 0,33 & 0,09 \\
\hline 2006 & 12,85 & 5,49 & 5,25 & 1,30 & 0,81 & 8,95 & 8,17 & 2,24 & 1,50 & 2,02 & 2,60 & 0,53 & 0,13 \\
\hline 2007 & 13,31 & 5,51 & 5,30 & 1,50 & 1,00 & 8,44 & 8,02 & 2,89 & 1,81 & 2,59 & 2,85 & 0,40 & 0,17 \\
\hline 2008 & 12,50 & 5,67 & 5,11 & 1,11 & 0,61 & 9,42 & 8,65 & 2,20 & 1,11 & 1,97 & 2,00 & 0,18 & 0,11 \\
\hline 2009 & 8,82 & 3,80 & 3,79 & 0,58 & 0,65 & 6,11 & 5,94 & 1,07 & 1,26 & 1,47 & 1,64 & 0,19 & 0,07 \\
\hline 2010 & 8,69 & 3,71 & 3,86 & 0,68 & 0,44 & 5,83 & 6,01 & 1,38 & 0,89 & 1,60 & 1,86 & 0,08 & 0,00 \\
\hline 2011 & 7,79 & 3,71 & 2,92 & 0,63 & 0,53 & 6,10 & 4,37 & 1,11 & 1,05 & 1,39 & 1,65 & 0,26 & 0,00 \\
\hline 2012 & 7,57 & 3,30 & 3,04 & 0,63 & 0,59 & 5,30 & 4,32 & 1,17 & 1,06 & 1,35 & 1,75 & 0,20 & 0,12 \\
\hline 2013 & 6,10 & 2,41 & 2,79 & 0,52 & 0,38 & 3,84 & 4,28 & 0,88 & 0,74 & 0,94 & 1,47 & 0,20 & 0,03 \\
\hline 2014 & 7,18 & 3,19 & 2,97 & 0,60 & 0,41 & 4,44 & 4,76 & 1,11 & 0,76 & 1,98 & 1,33 & 0,15 & 0,07 \\
\hline 1996-2008 & 14,56 & 6,25 & 5,87 & 1,72 & 0,72 & 10,16 & 9,43 & 3,09 & 1,35 & 2,45 & 2,72 & 0,55 & 0,09 \\
\hline 2009-2014 & 7,69 & 3,35 & 3,23 & 0,61 & 0,50 & 5,27 & 4,95 & 1,12 & 0,96 & 1,46 & 1,62 & 0,18 & 0,05 \\
\hline
\end{tabular}


Source: Database of the Statistical Office of the Slovak Republic (SOSR 2015)

$\mathrm{Km}=$ kilometers

MV= Motor vehicle $=$ all other-than-motorcycle-motor vehicle users

Ped $=$ Pedestrians

Bic $=$ Bicycle

$\mathrm{MC}=$ Motorcycle users 
Table A2. Age-standardized mortality rates per 100,000 person years for total population, males and females, individual age groups in the Slovak Republic in 1996-2014.

\begin{tabular}{|c|cccc|ccccccccc|c|}
\hline & \multicolumn{9}{|c}{ Total } & \multicolumn{3}{c|}{ Males } & \multicolumn{4}{c|}{ Females } \\
year & $<15$ & $15-24$ & $25-64$ & $65+$ & All & $<15$ & $15-24$ & $25-64$ & $65+$ & All & $<15$ & $15-24$ & $25-64$ & $65+$ \\
\hline 1996 & 4,60 & 16,45 & 17,73 & 23,52 & 24,93 & 5,07 & 26,16 & 30,25 & 37,45 & 6,50 & 4,10 & 6,41 & 5,69 & 15,32 \\
1997 & 3,93 & 24,12 & 21,71 & 25,85 & 31,64 & 5,00 & 38,90 & 37,41 & 47,85 & 6,69 & 2,80 & 8,80 & 6,71 & 11,68 \\
1998 & 3,97 & 22,45 & 22,58 & 31,06 & 32,85 & 4,02 & 34,76 & 39,36 & 56,70 & 7,26 & 3,93 & 9,67 & 6,53 & 14,39 \\
1999 & 5,22 & 16,81 & 17,95 & 22,88 & 25,25 & 7,18 & 26,83 & 30,45 & 34,31 & 6,08 & 3,18 & 6,41 & 5,94 & 12,12 \\
2000 & 4,21 & 17,31 & 16,69 & 22,67 & 24,85 & 6,78 & 26,05 & 29,19 & 38,55 & 5,28 & 1,52 & 8,24 & 4,79 & 11,41 \\
2001 & 4,09 & 15,35 & 16,75 & 19,26 & 23,20 & 6,02 & 23,61 & 28,45 & 31,73 & 5,41 & 2,08 & 6,74 & 5,51 & 9,90 \\
2002 & 3,60 & 18,09 & 14,82 & 18,47 & 21,34 & 4,30 & 26,51 & 24,32 & 34,54 & 5,88 & 2,87 & 9,33 & 5,65 & 8,57 \\
2003 & 2,66 & 16,02 & 17,92 & 17,85 & 23,12 & 2,90 & 23,76 & 29,54 & 31,83 & 6,21 & 2,42 & 7,97 & 6,75 & 8,93 \\
2004 & 4,06 & 14,39 & 15,56 & 16,64 & 21,69 & 4,49 & 22,78 & 26,38 & 32,07 & 5,14 & 3,62 & 5,64 & 5,08 & 7,84 \\
2005 & 2,49 & 13,93 & 15,49 & 16,68 & 20,23 & 2,31 & 21,79 & 25,62 & 28,08 & 5,53 & 2,68 & 5,74 & 5,86 & 9,40 \\
2006 & 1,90 & 15,53 & 15,71 & 17,59 & 20,86 & 2,04 & 25,52 & 25,88 & 28,37 & 5,29 & 1,75 & 5,10 & 5,87 & 9,79 \\
2007 & 3,74 & 13,45 & 15,79 & 20,30 & 21,16 & 4,93 & 20,28 & 25,48 & 33,90 & 6,02 & 2,49 & 6,33 & 6,38 & 10,92 \\
2008 & 3,29 & 16,75 & 14,35 & 16,60 & 21,39 & 4,12 & 28,05 & 24,73 & 31,35 & 4,25 & 2,43 & 4,98 & 4,23 & 7,08 \\
2009 & 1,33 & 11,14 & 10,61 & 12,25 & 14,39 & 1,89 & 18,79 & 17,72 & 17,76 & 3,37 & 0,74 & 3,18 & 3,68 & 7,40 \\
2010 & 2,26 & 11,79 & 9,89 & 11,84 & 14,11 & 2,99 & 18,48 & 16,42 & 19,70 & 3,55 & 1,48 & 4,81 & 3,46 & 6,46 \\
2011 & 1,66 & 10,64 & 9,09 & 10,13 & 12,62 & 1,66 & 16,35 & 15,00 & 18,35 & 3,30 & 1,66 & 4,70 & 3,26 & 4,98 \\
2012 & 1,30 & 9,97 & 8,99 & 10,17 & 11,85 & 1,39 & 15,13 & 14,17 & 17,41 & 3,43 & 1,21 & 4,61 & 3,83 & 4,46 \\
2013 & 0,71 & 7,07 & 7,35 & 9,62 & 9,74 & 0,92 & 11,61 & 11,62 & 15,97 & 2,65 & 0,49 & 2,32 & 3,10 & 5,21 \\
2014 & 0,94 & 9,76 & 8,58 & 9,59 & 11,06 & 1,14 & 13,25 & 13,46 & 16,61 & 3,53 & 0,73 & 6,13 & 3,71 & 4,90 \\
\hline
\end{tabular}

Source: Database of the Statistical Office of the Slovak Republic (SOSR 2015) 